\title{
VOF Simulation of The Cavitating Flow in High Pressure GDI Injectors
}

\author{
F. Giussani ${ }^{1 *}$, A. Montorfano ${ }^{1}$, F. Piscaglia ${ }^{1}$, A. Onorati ${ }^{1}$, J. Hélie $^{2}$ \\ ${ }^{1}$ Dip. di Energia, Politecnico di Milano, via Lambruschini 4, I-20156 Milan (Italy) \\ ${ }^{2}$ Continental Automotive SAS, 1 av. Paul Ourliac BP 1149, 31036 Toulouse Cedex 1 (France) \\ *Corresponding author: filippo.giussani@polimi.it
}

\begin{abstract}
The paper describes the development in the OpenFOAM ${ }^{\circledR}$ technology of a dynamic multiphase Volume-of-Fluid (VoF) solver, supporting mesh handling with topological changes, that has been used for the study of the physics of the primary jet breakup and of the flow disturbance induced by the nozzle geometry during the injector opening event in high-pressure Gasoline Direct Injection (GDI) engines. Turbulence modeling based on a scale-resolving approach has been applied, while phase change of fuel is accounted by means of a cavitation model that has been coupled with the VOF solver. Simulations have been carried out on a 6-hole prototype injector, especially developed for investigations in the framework of the collaborative project FUI MAGIE and provided by Continental Automotive SAS. Special attention has been paid to the domain decomposition strategy and to the code development of the solver, to ensure good load balancing and to minimize inter-processor communication, to achieve good performance and also high scalability on large computing clusters.
\end{abstract}

\section{Keywords}

Volume-of-fluid, GDI injectors, topologically changing mesh, hybrid RANS/LES, cavitation, OpenFOAM ${ }^{\circledR}$

\section{Introduction}

Turbulence certainly has a direct impact on thermodynamic efficiency, brake power and emissions of the engine, since its influence extends from volumetric efficiency to air/fuel mixing, combustion and heat transfer. In a gasoline engine, there are two strategies for injecting the fuel: Port Fuel Injection (PFI), a well known technology to favor air/fuel mixing, and Gasoline Direct Injection (GDI), which consists in injecting the fuel directly into the cylinder. GDI is becoming a common solution in automotive industry since it allows for a great flexibility in the air/fuel ratio, leading to low fuel consumption and emissions at light loads (lean or stratified mode) and high power output during rapid accelerations and heavy loads (power mode) [1,2]. These different conditions are not only related to the amount of fuel injected into the combustion chamber, but also to the way the injection is performed: level of atomization, penetration and diffusion of fuel. Experimental campaigns showed that spray formation is mainly influenced by the geometry of the injector itself (L/D ratio, number of holes, internal geometry, etc.) [3, 4], the operating pressure and the needle lift curve [5]. Fuel is characterized by high velocities (order of hundreds of meters per second) and reduced timescales, so that time-transient phenomena play an important role. Moreover, because of the strong acceleration of the liquid phase inside the injector nozzle, pressure may drop below the saturation value causing the onset of cavitation, which strongly modifies the internal flow field due to the presence of bubbles. Hence, a better comprehension of spray characteristics could help to improve engine performance and injector design. Simulating the injector opening event and the spray primary breakup are still on the frontier of modern modeling science. Since primary breakup is strictly related to turbulence inside the injector, a correct way to describe turbulence is crucial for a successful simulation. In this sense, a time-resolved approach like seems the most appropriate to model turbulence in injectors and also a straightforward way to overcome the intrinsic lack of time resolution of the Unsteady Reynolds-Averaged Navier-Stokes (URANS) equations [6]. Disperse sprays are usually simulated by a Lagrangian/Eulerian approach [7]: particles reside in the Eulerian grid cells and introduce source terms in the Eulerian conservation laws to account for interactions between the two phases. To simulate fuel injection, the continuous liquid core in the near-nozzle region is modeled by discrete parcels which typically are assumed to have the same characteristic size as the nozzle exit diameter and phenomenological models are used to account for breakup [8], as well as collision and coalescence of liquid droplets [9]. The above mentioned method requires a precursor nozzle flow simulation of the Eulerian liquid phase to predict the flow conditions at the nozzle exit [10]. The coupling between the internal and the external nozzle simulation is inherently weak, due to potential inconsistencies of the two-phase models in the two separate simulations [10]. On the other hand, engine sprays, and sprays in general, could be better described using a continuum for both the liquid and the gas phases, where conservation laws are solved under Eulerian flow assumptions and grid is refined until its resolution allows for solving droplets or bubbles without introducing any conceptual particles. In such a case, a transport equation for an indicator function is used to track the liquid-gas interface $[11,12,13]$. In this work the Volume-of-fluid (VOF) approach is adopted: governing equations are solved for a fluid whose thermophysical properties are a weighted average of the liquid and the vapor phases according to the liquid fraction. Additional terms are inserted in the equations to account for the presence of a physical interface. See Sec. for more details. Besides, since pressure inside the injector nozzle may drop below saturation pressure, a source term for cavitation in the momentum and continuity equations has been included. 
Finally, a mesh motion strategy based on topological changes $[14,15,16]$ has been coupled with the VOF solver and applied to simulate the needle movement: the aim was to study the early stage of the cavitating flow in the internal nozzle at the injector opening, where the continuum hypothesis is assumed to be valid. Some simplifying assumptions have been introduced in the present version of the solver, which represents an initial step towards a more comprehensive framework for injectors simulation: the fluid behavior can be regarded as incompressible and all gaseous phases (air + fuel vapor) can be represented by only one component (i.e., the fuel vapor). The developed code in the OpenFOAM technology has been tested on large supercomputers at the Argonne National Laboratory. New algorithms to perform load-balanced constrained decomposition, compatible with topological changes, have been developed.

\section{Test case}

The selected test case chosen for validation and testing of the dynamic VOF solver is a 6-hole prototype injector prototype provided by Continental (Fig. 1, left), especially developed for investigations in the framework of the collaborative project FUI MAGIE. Each nozzle is cylindrical with diameter $d=182 \mu \mathrm{m}$ and length $L=210 \mu \mathrm{m}$. Needle lift measurements (Fig. 1, right) were made available by Dr. Jin Wang, at Argonne National Laboratory, through X-ray propagation-based phase-enhanced micro-imaging [17].
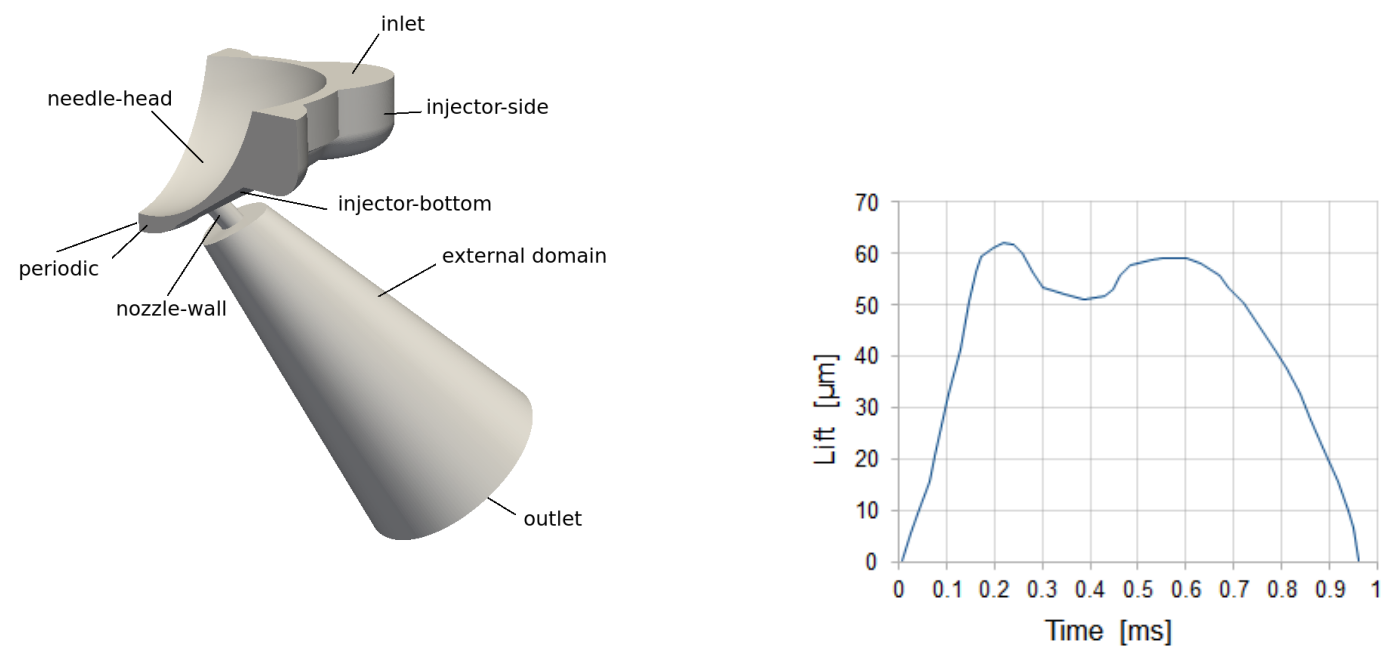

Figure 1. 6-hole prototype injector; only $1 / 6$ of the real component has been simulated (left). Injector lift curve (right).

In the experiments, conditions of liquid gasoline were $T=293 \mathrm{~K}$ and $p=30$ bar. Fuel was injected at ambient temperature in a large transparent chamber filled with air at ambient pressure and temperature. When the static pressure inside the nozzle drops below the vaporization pressure of the fuel, the liquid starts to cavitate and turns into the gaseous state of the fuel. Under these conditions, three phases of the fluid are observed: air, fuel vapor and liquid.

\section{Two-phase VOF solver and cavitation model}

A two-phase dynamic (isothermal and immiscible) VOF incompressible solver, including a cavitation model, has been used to simulate the injector opening event; in the simulations the liquid is assumed to be injected into a plenum chamber. The dynamic VOF solver includes a transport equation for the liquid fraction $\alpha_{l}$ (Eq. (1)), that is defined as the ratio of the liquid volume contained in a cell to the total liquid volume. The conservation equation for $\alpha_{l}$ is:

$$
\frac{\partial \alpha_{l}}{\partial t}+\nabla \cdot\left(\alpha_{l}\left(\mathbf{U}-\mathbf{U}_{b}\right)\right)+\nabla \cdot\left[\alpha_{l}\left(1-\alpha_{l}\right) \mathbf{U}_{c}\right]=\frac{\dot{m}}{\rho_{l}}
$$

where $\dot{m}$ is the mass source due to cavitation and $\left(\mathbf{U}-\mathbf{U}_{b}\right)$ is the relative velocity field between the fluid and the moving boundary. From the definition of $\alpha$, it results that $\alpha_{l}+\alpha_{v}=1$ where subscripts $l$ and $v$ stand for liquid and vapor respectively. The volume fraction is used to scale the physical properties of the mixture as:

$$
\rho=\rho_{l} \alpha_{l}+\left(1-\alpha_{l}\right) \rho_{v} \text { and } \mu=\mu_{l} \alpha_{l}+\left(1-\alpha_{l}\right) \mu_{v}
$$

The weighted physical properties of mixture are then used in the momentum equation:

$$
\frac{\partial(\rho \mathbf{U})}{\partial t}+\nabla \cdot\left(\rho\left(\mathbf{U}-\mathbf{U}_{b}\right) \mathbf{U}\right)-\nabla \cdot \mu \nabla \mathbf{U}-\rho \mathbf{g}=-\nabla p-\mathbf{F}_{s}
$$


On the right-hand side, $\mathbf{F}_{s}$ is surface force defined as $\sigma \kappa(x) \mathbf{n}$ with curvature $\kappa(x)=\nabla \cdot \mathbf{n}$, interface normal face unit vector $\mathbf{n}=\nabla \alpha_{l} /\left|\nabla \alpha_{l}\right|$, and surface tension $\sigma$. The last term on the left-hand side of Eq. 1 is known as the artificial compression term and it is non-zero only at the interface due to $\alpha_{l}\left(1-\alpha_{l}\right)$. The compression term has the role of shrinking the phase-interface towards a sharper one [18]. The compression term does not bias the solution in any way and it only corrects the flux of $\alpha_{l}$ in the interface-normal direction. In order to enforce this procedure, Weller [19] suggested the compression velocity to be calculated as:

$$
\mathbf{U}_{c}=C_{\alpha}|\mathbf{U}| \mathbf{n}
$$

The intensity of the interface compression is controlled by a constant $C_{\alpha}$, which yields no compression when zero, a conservative compression for $C_{\alpha}=1$ and high compression for $C_{\alpha}>1$ [18]. On the right-hand side, the mass source for cavitation is modeled according to the theory by Schnerr and Sauer [20]. According to it, there are several vapor bubbles (or nuclei) inside the liquid, that act as the initial sources of the phase change; cavitation starts from their locations and due to their presence. The mass transfer rate between liquid and vapor phases can be defined as follows:

$$
\dot{m}=\alpha_{l} \dot{m}_{\alpha v}+\left(1-\alpha_{l}\right) \dot{m}_{\alpha c}
$$

where:

$$
\begin{aligned}
& \dot{m}_{\alpha c}=C_{c} \alpha_{l} \frac{3 \rho_{v} \rho_{l}}{R_{b} \rho} \sqrt{\frac{2}{3 \rho_{l}\left|p-p_{s a t}\right|}} \cdot \max \left(p-p_{s a t}, 0\right) \\
& \dot{m}_{\alpha v}=C_{v}\left(1+\alpha_{n}-\alpha_{l}\right) \frac{3 \rho_{v} \rho_{l}}{R_{b} \rho} \cdot \sqrt{\frac{2}{3 \rho_{l}\left|p-p_{s a t}\right|}} \min \left(p-p_{s a t}, 0\right)
\end{aligned}
$$

with $C_{c}$ and $C_{v}$ as, respectively, the condensation and vaporization rate coefficients chosen by the user. These coefficients represent the relaxation time that either phase needs to be transferred into the other one. According to previous works [21] $C_{v}$ and $C_{c}$ are chosen equal to 1, that equals to consider the same behavior for condensation and vaporization rate. Moreover the reciprocal bubble radius is defined as:

$$
\frac{1}{R_{b}}=\left[\frac{4}{3} \frac{\pi n \alpha_{l}}{\left(1+\alpha_{n}-\alpha_{l}\right)}\right]^{1 / 3}
$$

$\alpha_{n}=V_{n} / 1+V_{n}$ is the nuclei volume fraction; the latter is computed as:

$$
V_{n}=\frac{n \pi d_{n}^{3}}{6}
$$

with $n$ as the number of nuclei per unit volume and $d_{n}$ their diameter. Since to authors' knowledge there are no experiments about nucleation characteristics of liquid fuels, size and distribution of nuclei have been taken from experiments carried out with water. In this work, it is assumed a uniform nuclei distribution of $n=1.6 \cdot 10^{-8} \mathrm{~m}^{-3}$ with a diameter $d_{n}=2 \mathrm{~nm}$. Moreover, depending on the local properties of the flow, the extra diagonal terms in the linear system matrix of Eq. (1) can become very large (due to very high phase change rate), thus impairing the convergence rate. In order to improve the solution stability, the source term needs to be rewritten, so that Eq. 1 is formulated by decomposing the volume source $\dot{V}$ in an implicit and an explicit part:

$$
\dot{V}=\left[\frac{1}{\rho_{l}}-\alpha_{l}\left(\frac{1}{\rho_{l}}-\frac{1}{\rho_{v}}\right)\right] \dot{m}=A_{\alpha} \dot{m}=A_{\alpha}\left(\dot{m}_{\alpha v}-\dot{m}_{\alpha c}\right) \alpha_{l}+A_{\alpha} \dot{m}_{\alpha c}=\left(\dot{V}_{v}-\dot{V}_{c}\right) \alpha_{l}+\dot{V}_{c}
$$

the former being $\left(\dot{V}_{v}-\dot{V}_{c}\right)$, which enters the system matrix as a diagonal coefficient; on the other hand, the explicit part of the source term $\dot{V}_{c}$ will be written on the right-hand side of the linear system. The final form of the conservation equation of $\alpha_{l}$ (Eq. (11)) therefore reads:

$$
\frac{\partial \alpha_{l}}{\partial t}+\nabla \cdot\left(\alpha_{l}\left(\mathbf{U}-\mathbf{U}_{b}\right)\right)+\nabla \cdot\left[\alpha_{l}\left(1-\alpha_{l}\right) \mathbf{U}_{c}\right]=\left(\dot{V}_{v}-\dot{V}_{c}\right) \alpha_{l}+\dot{V}_{c}
$$

Turbulence has been accounted for by means of a Hybrid RANS/LES approach called Dynamic Length-Scale Resolution Model (DLRM) developed by the authors [22]. The underlying principle of DLRM is that the turbulent viscosity computed by a RANS model (the $k-\omega$ SST in this case) can be scaled down to Implicit LES when time and mesh resolution locally allow to solve turbulent structures. The momentum equation (3) is then complemented by the insertion of a turbulent stress tensor $\mathbf{B}=\mu_{t}\left(\nabla \mathbf{U}+\nabla^{T} \mathbf{U}\right)$ :

$$
\frac{\partial(\rho \mathbf{U})}{\partial t}+\nabla \cdot\left(\rho\left(\mathbf{U}-\mathbf{U}_{b}\right) \mathbf{U}\right)-\nabla \cdot \mu \nabla \mathbf{U}-\rho \mathbf{g}-\nabla \cdot \mathbf{B}=-\nabla p-\mathbf{F}_{s}
$$

whereas all other variables (including velocity) have now the meaning of partially-averaged quantities. A more detailed discussion on the physical significance of "partial average" can be found in e.g. [23]. 


\section{Dynamic mesh handling and parallel optimization}

The moving mesh functionality used in this work is described in $[14,15]$ and it has been applied as described in [16]. The injector opening event is simulated by dynamically attaching and detaching the conformal interface represented by the set of faces detachFaces in Fig. 2a, while a prescribed vertical motion is set for the boundary needleHead and for the corresponding moving cell set. Initially the first layer cell attached to bottomFaces and topfaces are respectively stretched and shrunk. Due to the very small gap between the injector needle and the injector body at closure $(O(\delta)=10 \mu \mathrm{m})$, the mesh handling could look particularly difficult: cell sizes change by orders of magnitude and this can impair the mesh smoothness in the near-nozzle region. To overcome this issue, when cell layers reach the $\mathrm{max} / \mathrm{min}$ layer thickness specified by the user, automatic addition/removal is triggered, ensuring constant cell quality during the whole simulation. In the needle region, the maximum thickness (triggering addition) was set to $6 \mu \mathrm{m}$, whereas the minimum threshold (triggering removal) was $0.35 \mu \mathrm{m}$.

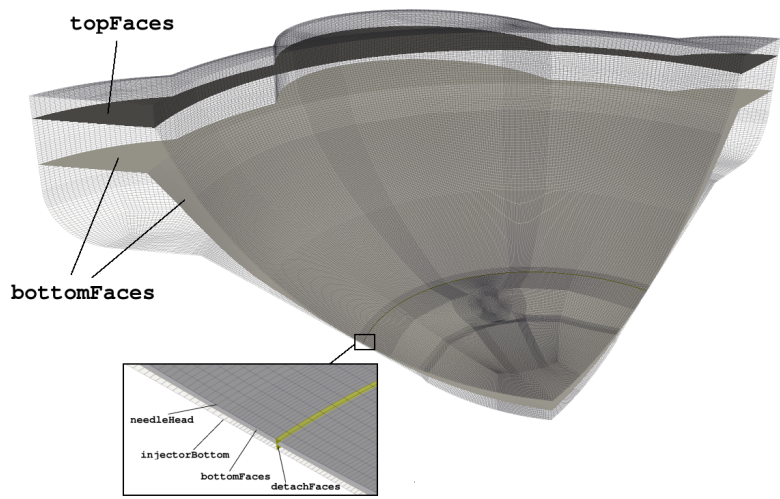

(a)

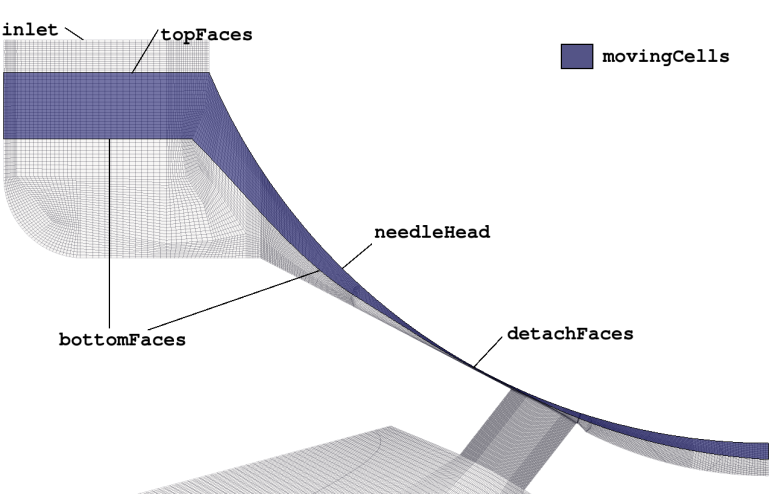

(b)

Figure 2. (a) Detail of face sets used by the solver: topFaces and bottomfaces are where layer A/R occurs, detachFaces are used to dynamically attach/detach the mesh; needleHead is the moving patch. (b) Cell sets used for decomposition

To run parallel simulations, the Finite Volume (FV) mesh has to be decomposed into a set of sub-domains, each to be assigned to a single core for processing. In simulations involving mesh motion with topological changes, new constraints in domain decomposition arise. In OpenFOAM ${ }^{\circledR}$, as well as in most of the CFD codes, topological changes cannot occur across inter-processor patches between neighboring sub-domains and the decomposition algorithm must be constrained in this sense. In other words, in regions where layer Addition/Removal (A/R) occurs, inter-processor faces cannot be parallel to layer $A / R$ surface as shown in Fig. $2 b$. Nevertheless, it is important for the decomposition to remain balanced despite the aforementioned restrictions; in addition, the algorithm should require minimal user intervention, allowing the automation of case setup for large simulation campaigns. A new strategy for mesh decomposition, which considers all the aforementioned aspects, has been developed and applied to the GDI injector. The decomposition algorithm proceeds as follows:

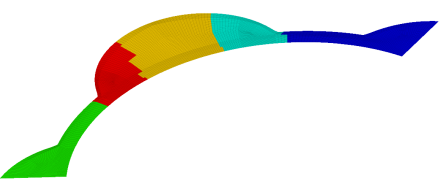

(a)

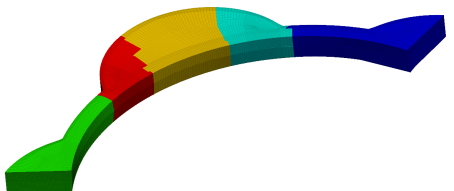

(b)

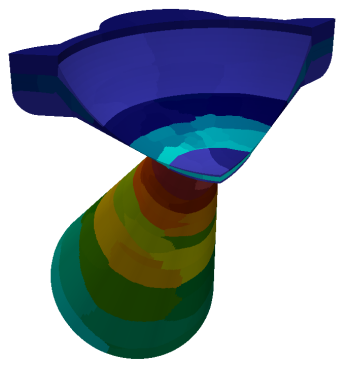

(c)

Figure 3. Overview of decomposition strategy: (a) Decomposition of face set topfaces using Scotch method; (b) Propagation of face set decomposition tho whole cell Set; (c) Complete decomposition

1. The grid is divided into several regions, in accordance with the decomposition constraints (layer $A / R$ zones, attach/detach zones, static parts Fig. 2b). Each mesh region is in turn decomposed into a number of subdomains, depending on the size of the region;

2. All face sets where dynamic layering is triggered (e.g. bottomFaces) are decomposed and subsets of faces are distributed among processors. For specified cell sets, cell decomposition is propagated perpendicularly 
to the face set using a cell-face walk algorithm, over the entire mesh region where layer addition/removal can be triggered, as shown in Figs. 3a-3b;

3. The remaining cells of the mesh are distributed among processors using non-constrained decomposition Fig. $3 c$

The decomposition strategy outlined above is very flexible, allowing for an almost perfect cell distribution (load balancing) over the processors with complex geometries and topological changes. The domain decomposition and overall balancing of the mesh used in this work is shown in Fig. 4. For similar geometries, the steps for domain decomposition can be easily automated, thus enabling automatic case setup in optimization/validation campaigns. Scalability tests were performed with the same solver on a simplified test case. Results are shown in Fig. 5. Three meshes with progressive refinement were tested, i.e., with $1 \mathrm{M}, 8 \mathrm{M}$, and $64 \mathrm{M}$ cells. Speedup is linear at least for a certain number of sub-domains per each mesh. The reason for the degradation of parallel performance over a certain threshold is still under investigation. However, since the present case has a number of cells that is very similar to one mesh used in the test (respectively $11 \mathrm{M}$ cells vs. 8M cells), a decomposition over 256 sub-domains has been chosen. Moreover, the same tests [24, 25] proved that using Preconditioned Conjugate Gradient (PCG) method instead of Geometric-Algebraic Multi-Grid (GAMG) to solve Poisson equation for pressure, can reduce the overall wall clock time but does not affect the scalability behavior, especially when a large amount of cores are used. On the other hand, there is also a chance that the performance decay is caused by a different convergence behavior of some sub-domains rather than an increased communication overhead. In case the interface-tracking resolution is delegated only to a few processors out of many, the consequent increased difficulty in solving the Poisson Equation (because of sharp pressure and density changes across the liquid/gas interface) on those few sub-domains can act as a bottleneck on the overall clock time. As a consequence, the parallel performance of the run might be impaired.

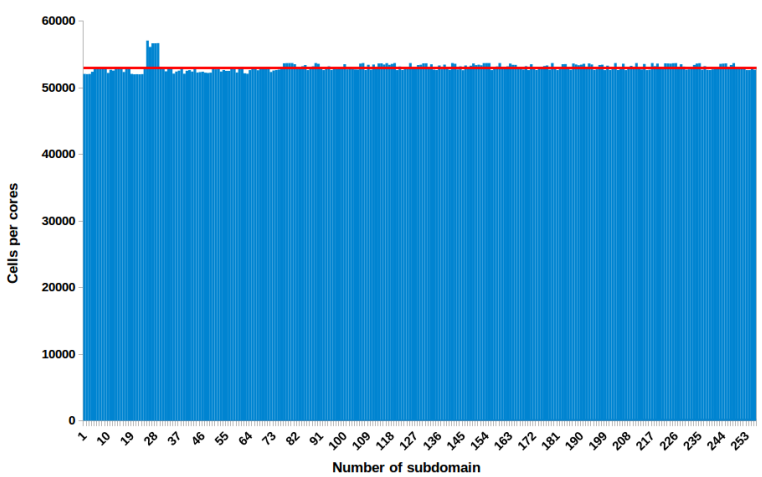

Figure 4. Histogram of number of cells per core. Red line represents mean value

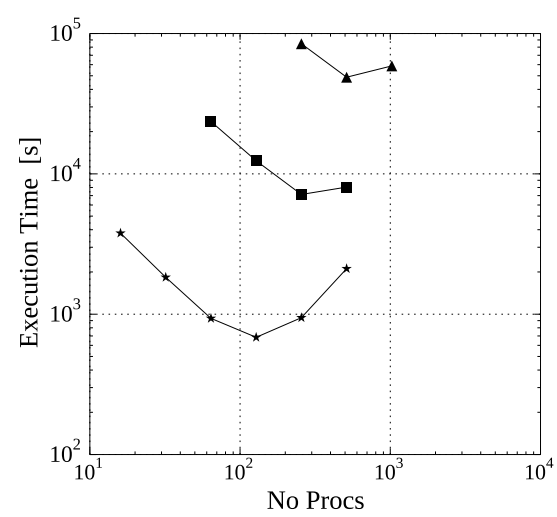

Figure 5. Left: Wall-clock time vs number of sub-domains. Right: Wall-clock time vs cells per processor. $-\star-1 \mathrm{M}$ mesh $-\mathbf{-}-8 \mathrm{M}$ mesh , $-\mathbf{\Delta}-64 \mathrm{M}$ mesh. Simulation time is $0.05 \mathrm{~s}$ for $1 \mathrm{M}$ and $8 \mathrm{M}$ cases, $0.025 \mathrm{~s}$ for $64 \mathrm{M}$ case.

\section{Case Setup \\ Geometry}

The computational mesh is of the order $11 \mathrm{M}$ cells ( $3 \mathrm{M}$ cells within the injector domain and $8 \mathrm{M}$ cells within the ambient) that allows a spatial resolution in the range $0.3-5 \mu \mathrm{m}$ (within the injector seat-nozzle) to $20 \mu \mathrm{m}$ (within the ambient domain). The mesh presents a maximum non-orthogonality of 76 degrees in the near-needle region, that forces the adoption of a limited scheme for laplacian discretization, together with at least 2 non-orthogonal correctors in the Poisson equation. Skewness maximum value it is around 1.35 which fulfills the good quality requirements. The injection chamber has been approximated by a conical volume (about $135 \mathrm{~mm}^{3}$ ), to limit the overall number of cells while keeping the lateral outlet patches sufficiently far not to interfere with the internal flow. Its length, along nozzle axis direction, is about 14 nozzle diameters, and its outlet diameter is about 10 nozzle diameters. From the previous work of Lu [21] it has been seen that such geometry approximation coupled with non-reflecting outlet condition [26] does not influence break-up phenomena. The block-structured, fully hexahedral FV mesh has 11M cells at injector closure. The approach chosen for turbulence modeling allows for the use of an increased cell size in the external region, where velocities are lower and turbulence dynamics are less critical for the global solution, limiting the overall number of cells. On the other hand, where cavitation occurs and a large amount of turbulence is generated, highly-resolved meshes are required to accurately capture the physics. Because of the CourantFriedrichs-Lewy (CFL) criterion, this greatly limits the size of time steps. n-Heptane has been chosen to simulate the fuel since its properties approximate the behavior of gasoline quite well [27]. 


\section{Numerical setup}

Time derivatives have been approximated by a second-order backward Euler scheme. Extensive development was done to preserve second order temporal accuracy with dynamic addition of cell layers in OpenFOAM [16, 28]. Pressure-velocity coupling is achieved by means of a transient-SIMPLE algorithm. The phase-fraction equation (Eq. (1)) is solved explicitly at the beginning of each time iteration by the Multi Universal Limiter for Explicit Solution (MULES) [29], a Flux Corrected Transport method developed by Weller to ensure boundedness of the $\alpha$ flux on all cell faces, which requires CFL to be lower than 1. In addition, special Total Variation Diminishing (TVD) methods are used for the compression velocity term $\nabla \cdot\left[\alpha_{l}\left(1-\alpha_{l}\right) \mathbf{U}_{c}\right]$ and $\nabla \cdot\left(\mathbf{U} \alpha_{l}\right)$. The former has been discretized with interface Compression scheme [30], while the latter with van Leer, as suggested by Weller [19]. For the convective term $\nabla \cdot(\phi \mathbf{U})$ a Linear Upwind with Stabilized Transport (LUST) scheme has been selected [31], while pure second order spatial discretization has been used for transport term of turbulent variables. A total-pressure boundary condition has been applied on the inlet section, and normal velocity is indirectly obtained from the pressure gradient on the boundary. Moreover, the sac domain has been initialized as containing only liquid fuel, for purging of fuel at considered pressure is not significant. Turbulence is mainly generated by the fluid/wall interaction in the needle region, and by the shear layer at the nozzle outlet, thus no synthetic turbulence generation has been deemed as required at the inlet. This allowed a reduction in the computational cost of about $10 \%$.
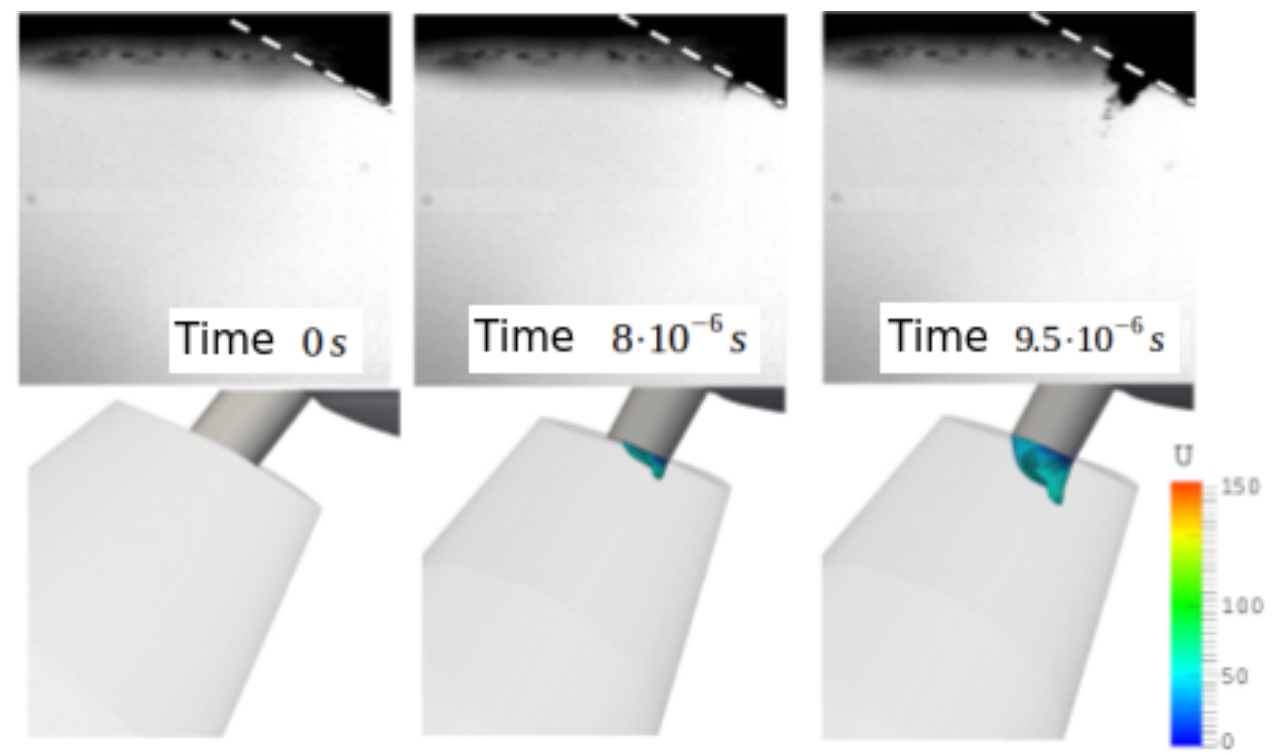

Figure 6. Comparison of experiments (top) and simulation (bottom) at early stage of injection.

\section{Injector opening: preliminary results}

Since the simulation is very demanding in terms of computational resources, only partial results are available at the time this paper is written. Fig. 6 shows the evolution of $n$-Heptane spray inside the combustion chamber up to $0.095 \mathrm{~ms}$ After Start Of Injection (ASOI). Simulation results (isosurface of liquid fraction at $\alpha=1$ ) is compared with high-speed shadowgraphs. The agreements in terms of shape and size of the jet is very good. As the needle starts to open, the nozzle is filled progressively with a non-uniform distribution of liquid due to the high level of turbulence; therefore, the tip of the spray jet does not have the typical mushroom shape described by other authors [21, 32], who analyzed the primary breakup at fixed needle position (maximum lift). This mechanism can be seen in Fig. 7 where the nozzle injector has been cut to show the internal flow. By looking at the constant pressure line at $p=p_{\text {sat }}$ in Fig. 7, the main cavitation region can be detected in correspondence of the vena contracta between the needle sac and the nozzle entry. A recirculation vortex is formed in this point, where pressure drops below the saturation value. The pocket of vapor is then transported towards the jet core and it is disrupted by the turbulent structures that reside there. A clearer idea of the turbulent structures that are formed in the nozzle can be observed in Fig. 8. Similarly to [21], a pair of counter-rotating vortices, roughly aligned with the nozzle axis, is formed in the jet core together with finer turbulent structures responsible of the instability of the jet (Fig. 8). The pressure drop occurring at the vortex cores causes again the fuel to cavitate, and elongated vapor regions are formed along the nozzle axis. This phenomenon is clearly visible in Fig. 9 where an iso-line of pressure at $p_{\text {sat }}$ is superposed to a contour plot of liquid phase fraction. The aforementioned vortex pair can be also the cause of the symmetric shape of the jet tip observed in Figs. 6 and 7. Finally, hints of primary breakup can be observed in Fig. 8: the development of some unstable structures like instability waves on the upper side of the jet and wrinkling of the liquid front as it enters the injection chamber are clearly visible. 


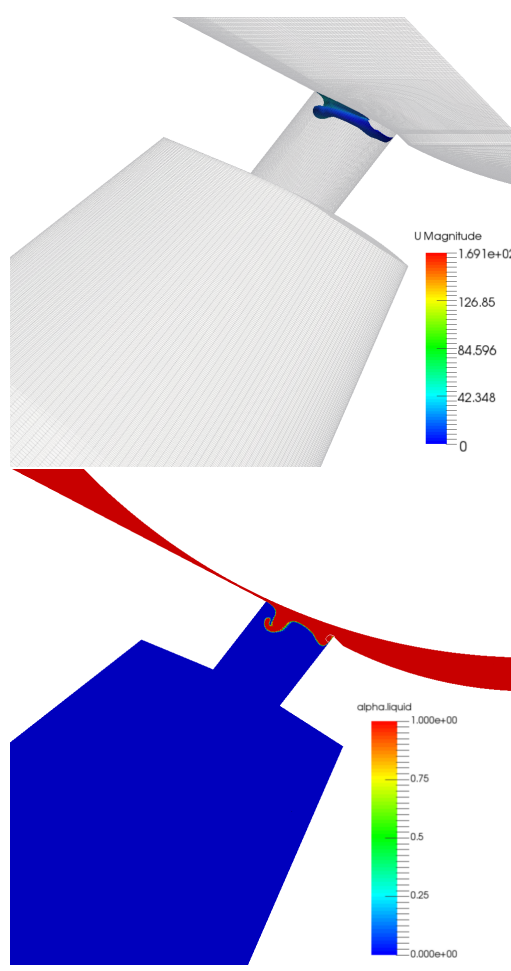

(a) $t=0.001 \mathrm{~ms}$

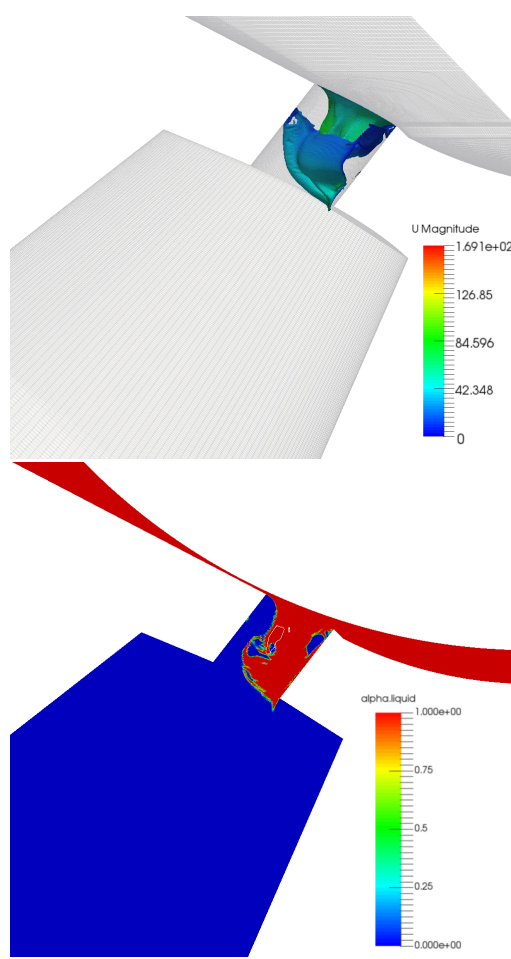

(d) $t=0.0065 \mathrm{~ms}$

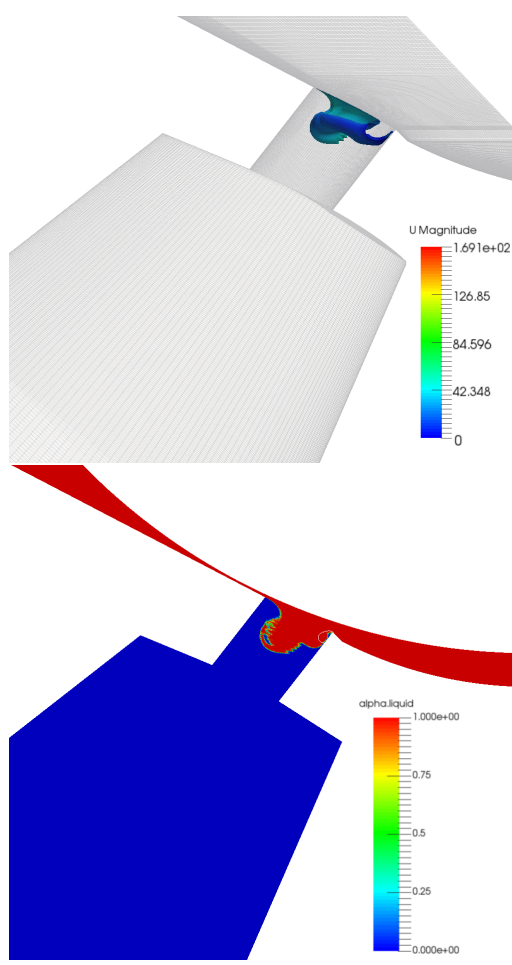

(b) $t=0.0025 \mathrm{~ms}$

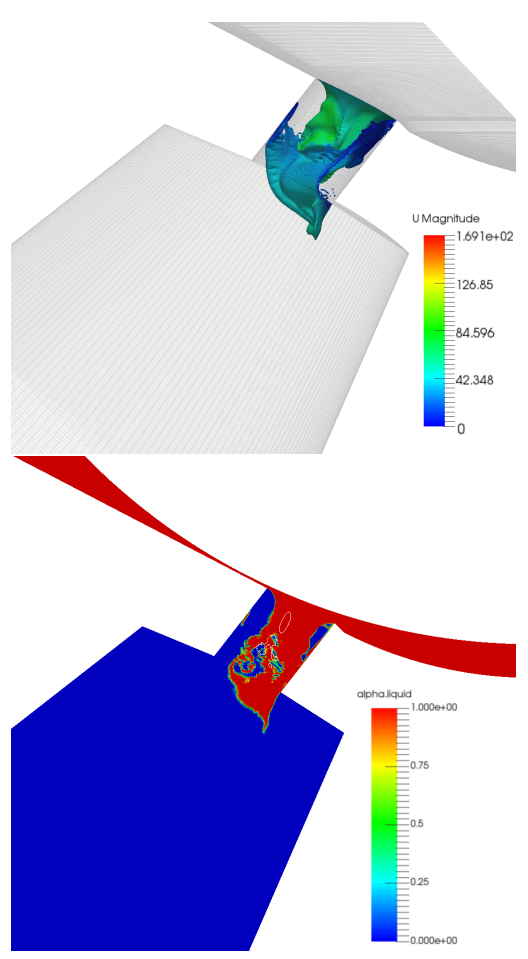

(e) $t=0.008 \mathrm{~ms}$

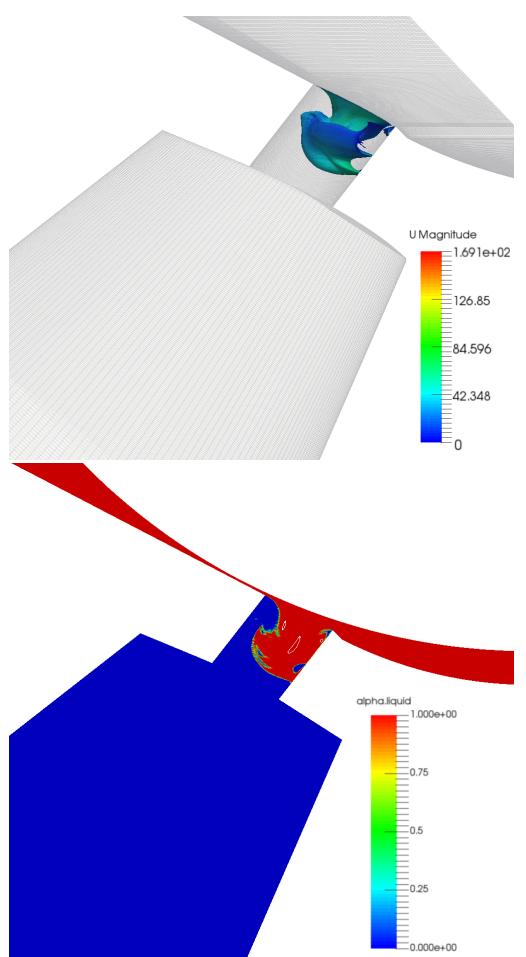

(c) $t=0.005 \mathrm{~ms}$
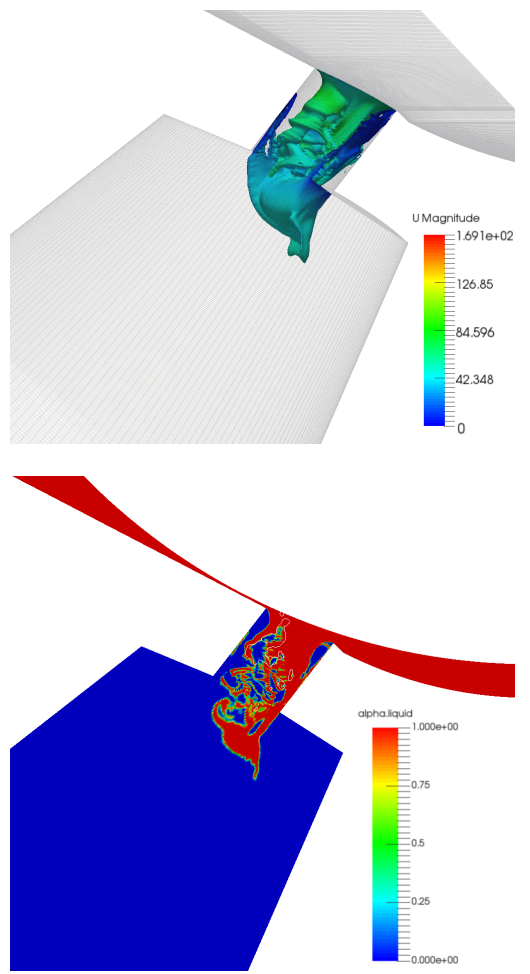

(f) $t=0.01 \mathrm{~ms}$

Figure 7. Sequence of snapshots of the spray exiting the nozzle following SOI. Top row: isosurface with $\alpha_{l}=0.5$, bottom row: sliced view of the above, with constant pressure line at $p=p_{\text {sat }}$. 

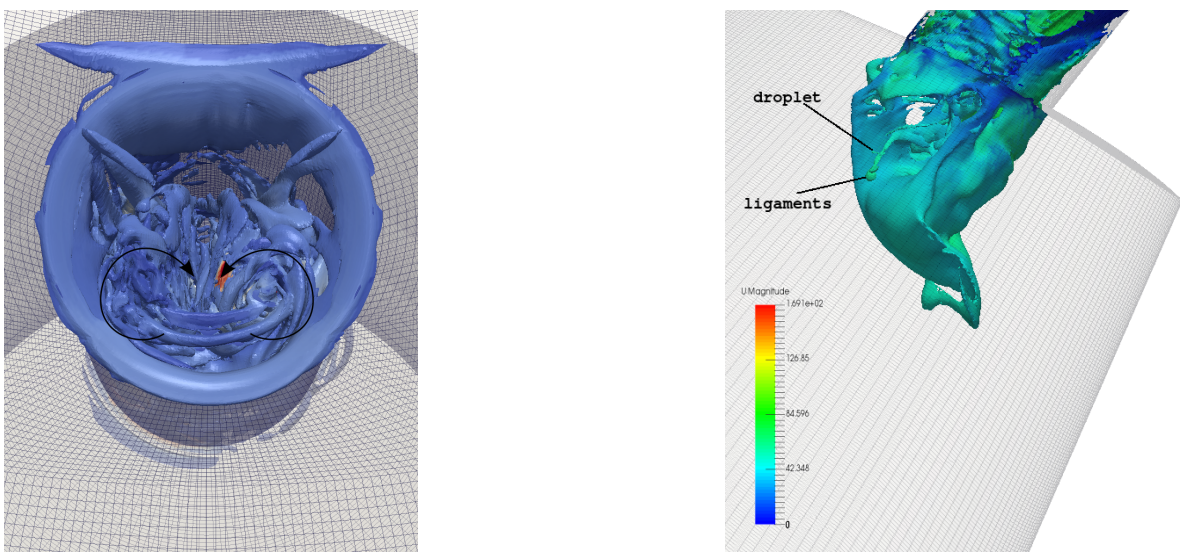

Figure 8. Left: vortical structures (isosurface of $Q=10^{4}$ ) in the nozzle at $t=9.5 \cdot 10^{-6} \mathrm{~s}$; right: liquid-vapor interface
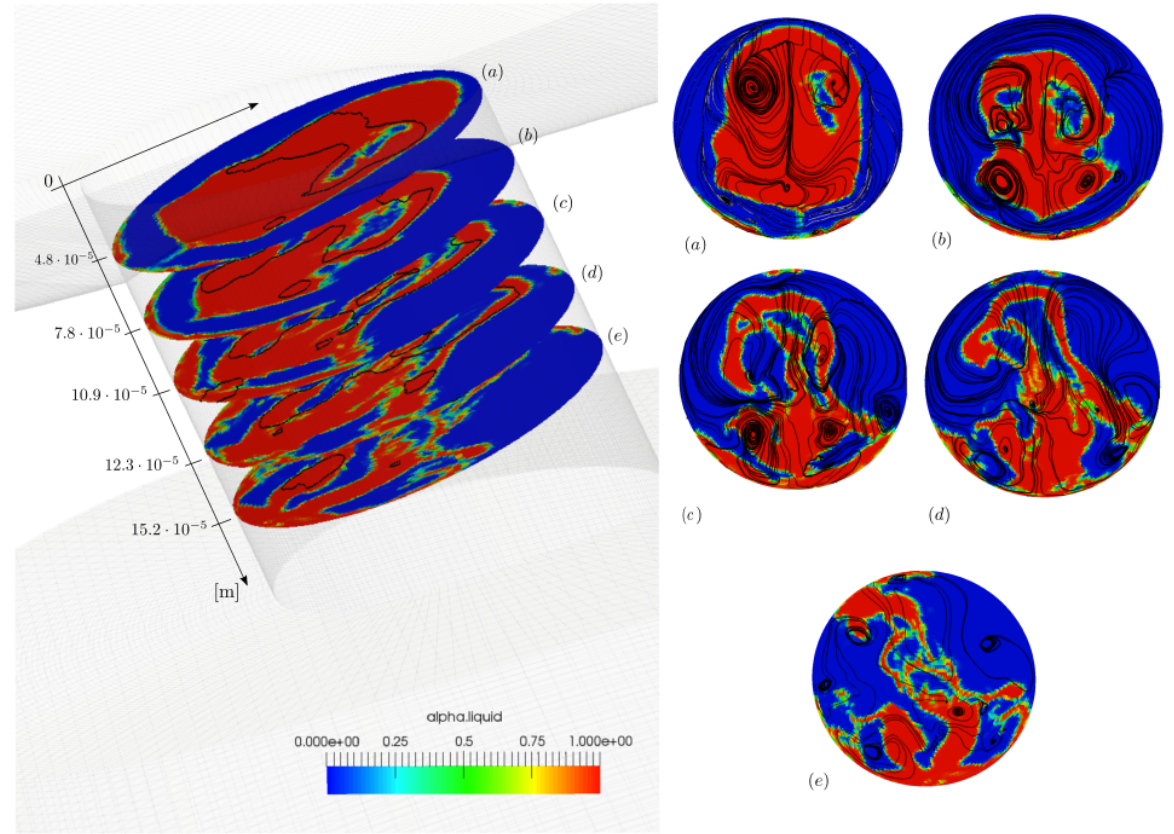

(c)

(d)

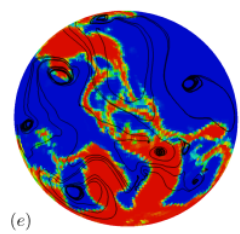

Figure 9. Horizontal cuts inside the nozzle at different height from nozzle inlet at time $9.5 \cdot 10^{-6} \mathrm{~s}$. The thin black line represents iso-contour of $p$ at $p_{\text {sat }}=4720 \mathrm{~Pa}$

\section{Conclusions}

The paper shows current developments done on dynamic simulation of multi-phase flows of GDI injectors, with particular focus on the coupling of an incompressible two-phase VOF solver with the cavitation model in a framework where the dynamic mesh based on topological changes is automatically handled in parallel. The solver has been applied to the simulation of a gasoline injector specifically designed for GDI engines and provided by Continental Automotive SAS. The case has been set up to simulate the early stages of the gasoline injection, using topological changes to implement mesh motion during injector opening. Details of the flow structures during the primary breakup are detected, which are in accordance with the findings of [21]. In this sense, the approach can be considered as promising and worthy of further investigation to assess its applicability during the design stage of injectors, with a particular focus on cavitation behavior. In particular, the initial assumptions about the incompressible nature of the flow have to be investigated, along with any effect arising from the consideration of only two gaseous phases. The solver also proved to be very stable: boundedness of the liquid fraction $\alpha_{l}$ was always preserved during the simulations even in presence of topological changes, so the method can be assumed to be fully conservative. Also, second-order accuracy was preserved both in space and time, which is not trivial in presence of dynamic topologically changing grids. Finally, a new decomposition method enabled good scalability of the code on large supercomputers. The developed code presented in this paper uses the most recent versions of OpenFOAM released by the OpenFOAM ${ }^{\circledR}$ Foundation and by OpenCFD ${ }^{\circledR}$ as development base frameworks. 


\section{Acknowledgments}

Authors would like to kindly thank the LCRC (Laboratory Computing Resource Center), Argonne National Lab, for making available the computing resources through the HPC cluster Blues within the PETSC-FOAM project.

\section{Nomenclature}

$T \quad$ temperature $[\mathrm{K}]$

$P \quad$ pressure [bar]

$\alpha_{l} \quad$ liquid volume fraction [-]

$t \quad$ time [s]

$\mathbf{U} \quad$ velocity [ $\mathrm{m} \mathrm{s}^{-1}$ ]

$\mathbf{U}_{b} \quad$ boundary velocity [ $\mathrm{m} \mathrm{s}^{-1}$ ]

$\mathbf{U}_{c} \quad$ compression velocity [ $\mathrm{m} \mathrm{s}^{-1}$ ]

$\alpha_{v} \quad$ vapor volume fraction [-]

$\rho_{l} \quad$ liquid density $\left[\mathrm{kg} \mathrm{m}^{-3}\right]$

$\rho \quad$ mixture density $\left[\mathrm{kg} \mathrm{m}^{-3}\right]$

$\rho_{V} \quad$ vapor density $\left[\mathrm{kg} \mathrm{m}^{-3}\right]$

$\mu \quad$ dynamic viscosity of mixture [Pa s]

$\mu_{l} \quad$ liquid dynamic viscosity [Pa s]

$\mu_{v} \quad$ vapor dynamic viscosity [Pa s ]

g gravity acceleration $\left[\mathrm{m} \mathrm{s}^{-2}\right]$

$\mathbf{F}_{s} \quad$ surface forces $\left[\mathrm{N} \mathrm{m}^{-2}\right]$

$\sigma \quad$ surface tension forces $\left[\mathrm{Nm}^{-1}\right]$

\begin{tabular}{|c|c|}
\hline$\kappa(x)$ & interface curvature $\left[\mathrm{m}^{-1}\right]$ \\
\hline $\mathbf{n}$ & normal vector [-] \\
\hline$C_{\alpha}$ & compression velocity coefficient [-] \\
\hline$C_{c}$ & condensation coefficient [-] \\
\hline$R_{b}$ & bubble radius $\left[\mathrm{m}^{-1}\right]$ \\
\hline$p_{\text {sat }}$ & saturation pressure $[\mathrm{Pa}]$ \\
\hline$C_{v}$ & vaporization coefficient [-] \\
\hline$n$ & nuclei concentration $\left[\mathrm{m}^{-3}\right]$ \\
\hline$\alpha_{n}$ & nuclei volume fraction [-] \\
\hline$V_{n}$ & total volume of nuclei $\left[\mathrm{m}^{-3}\right]$ \\
\hline$d_{n}$ & nuclei diameter $[\mathrm{m}]$ \\
\hline$\dot{V}$ & alpha equation source term $\left[\mathrm{s}^{-1}\right]$ \\
\hline$\dot{V}_{v}$ & vaporization source term $\left[\mathrm{s}^{-1}\right]$ \\
\hline$\dot{V}_{c}$ & condensation source term $\left[\mathrm{s}^{-1}\right]$ \\
\hline B & turbulent stress tensor $\left[\mathrm{N} \mathrm{m}^{-2}\right]$ \\
\hline$\mu_{t}$ & turbulent dynamic viscosity [Pa s] \\
\hline
\end{tabular}

\section{References}

[1] F. Zhao, D. Harringotn, and M. Lai, Automotive Gasoline Direct-Injection Engines, 2002. http: //books.sae.org/r-315/

[2] F.-Q. Zhao, M.-C. Lai, and D. L. Harrington, "A review of mixture preparation and combustion control strategies for spark-ignited direct-injection gasoline engines," in SAE Technical Paper. SAE International, 021997. http://dx.doi.org/10.4271/970627

[3] W.-M. Ren and H. Sayar, "Influence of nozzle geometry on spray atomization and shape for port fuel injector," in SAE Technical Paper. SAE International, 03 2001. http://dx.doi.org/10.4271/2001-01-0608

[4] M. Skogsberg, P. Dahlander, R. Lindgren, and I. Denbratt, "Effects of injector parameters on mixture formation for multi-hole nozzles in a spray-guided gasoline di engine," in SAE Technical Paper. SAE International, 04 2005. http://dx.doi.org/10.4271/2005-01-0097

[5] X. Zhang, L. J., and J. Wang, "Effect of fuel and nozzle geometry on the off-axis oscillation of needle in diesel injectors using high-speed x-ray phase contrast imaging," Journal of Instrumentation, vol. 11, no. 05, p. C05015, 2016. http://stacks.iop.org/1748-0221/11/i=05/a=C05015

[6] R. Payri, B. Tormos, J. Gimeno, and G. Bracho, "The potential of large eddy simulation (les) code for the modeling of flow in diesel injectors," Mathematical and Computer Modelling, vol. 52, no. 7-8, pp. 1151 - 1160, 2010, mathematical Models in Medicine, Business \& amp; Engineering 2009. http://www.sciencedirect.com/science/article/pii/S089571771000097X

[7] K. John and Dukowicz, "A particle-fluid numerical model for liquid sprays," Journal of Computational Physics, vol. 35, no. 2, pp. 229-253, 1980. http://www.sciencedirect.com/science/article/pii/002199918090087X

[8] Reitz and D. Rolf, "Modeling atomization processes in high-pressure vaporizing sprays," Atomization and Spray technology, vol. 3, no. 4, pp. 309-337, 1987.

[9] A. Amsden, "A block-structured program for engines with vertical and canted valves," 1997.

[10] E. Giannadakis, M. Gavaises, and C. Arcoumanis, "Modeling of cavitation in Diesel injector nozzles," International Journal of Fluid Mechanics, vol. 616, pp. 153-193, 2008.

[11] T. Menard, S. Tanguy, and A. Berlemont, "Coupling level set/VOF/ghost fluid methods: Validation and application to 3D simulation of the primary break-up of a liquid jet," International Journal of Multiphase Flow, vol. 33, no. 5, pp. $510-524$, 2007. http://www.sciencedirect.com/science/article/pii/S0301932206001832

[12] H. Pitsch and O. Desjardins, "Deatailed umerical investigation of turbulent atomization of liquid jets," Atomization and Sprays, vol. 20, no. 4, pp. 311-336, 2010.

[13] S. S. Deshpande, M. F. Trujillo, X. Wu, and G. Chahine, "Computational and experimental characterization of a liquid jet plunging into a quiescent pool at shallow inclination," International Journal of Heat and Fluid Flow, vol. 34, no. 0, pp. 1 - 14, 2012. http://www.sciencedirect.com/science/article/pii/S0142727X12000197

[14] F. Piscaglia, A. Montorfano, and A. Onorati, "An extension of the dynamic mesh handling with topological changes for LES of ICE in OpenFOAM," SAE paper 2015-01-0384, 2015, SAE World Congress Exhibition, Detroit, Michigan (USA), 2015, http://dx.doi.org/10.4271/2015-01-0384. 
[15] F. Piscaglia, A. Montorfano, and A. Onorati, "Development of fully-automatic parallel algorithms for mesh handling in the OpenFOAM-2.2.x technology," SAE Technical Paper 2013-24-0027, 2013, http://dx.doi.org/10.4271/ 2013-24-0027.

[16] F. Piscaglia, A. Montorfano et al., "Hybrid RANS/LES of Moving Boundary Problems: Application to Cavitating Sprays and In-Cylinder Flows," in International Multidimensional Engine Modeling User's Group Meeting At the SAE Congress, April 11th 2016, https://imem.cray.com/agenda.html.

[17] W.-K. Lee, K. Fezzaa, and J. Wang, "Metrology of steel micronozzles using x-ray propagationbased phase-enhanced microimaging," Applied Physics Letters, vol. 87, no. 8, p. 084105, 2005. http://dx.doi.org/10.1063/1.2034099

[18] E. Berberovic, "Investigation of free-surface flow associated with drop impact: Numerical simulations and theoretical modeling," Ph.D. dissertation, Technische Universität, Darmstadt, November 2010. http://tuprints.ulb.tu-darmstadt.de/2319/

[19] H. Weller, "A new approach to vof-based interface capturing methods for incompressible and compressible flow," Technical Report TR/HGW/04,OpenCFD Ltd., Technical Report TR/HGW/04,OpenCFD Ltd., 2008.

[20] G. Schnerr and J. Sauer, "Unsteady cavitating flow - a new cavitation model based on a modified front capturing method and bubble dynamics." ASME Fluids Engineering Summer Meeting, June 2011-15, Boston (USA), 2000.

[21] N. Lu, F.-X. Demoulin, J. Reveillon, and J. Chesnel, "Large eddy simulation of cavitation and atomization in injector flows using openfoam," ILASS - Europe 2014, 26th Annual Conference on Liquid Atomization and Spray Systems, Bremen, Germany, 2014.

[22] F. Piscaglia, A. Montorfano, and A. Onorati, "A Scale Adaptive Filtering Technique for Turbulence Modeling of Unsteady Flows in IC Engines," SAE Int. J. Engines, Paper n. 2015-01-0395, 2015, http://dx.doi.org/10.4271/ 2015-01-0395.

[23] P. Sagaut, Large-Eddy Simulation for Incompressible Flows: an Introduction, ser. Scientific computation. Springer-Verlag, 2006.

[24] J. Hélie, J. Chesnel, and N. LU, "Parallel performance of OpenFOAM in industrial applications," PRACE project pa1507, Tech. Rep., 2015.

[25] A. Montorfano, F. Giussani, F. Piscaglia, and J. Hélie, "Parallel performance of OpenFOAM in industrial applications," PRACE project pa3053, Tech. Rep., 2016.

[26] F. Piscaglia, A. Montorfano, and A. Onorati, "Development of a non-reflecting boundary condition for multidimensional nonlinear duct acoustic computatio," Journal of Sound and Vibration, vol. 332, no. 4, pp. 922-935, 2013.

[27] D. L. Hung, D. L. Harrington, A. H. Gandhi, L. E. Markle, S. E. Parrish, J. S. Shakal, H. Sayar, S. D. Cummings, and J. L. Kramer, "Gasoline fuel injector spray measurement and characterization - a new sae j2715 recommended practice," SAE International Journal of Fuels and Lubricants, vol. 1, 042008. http://gen.lib.rus.ec/scimag/index.php?s=10.4271/2008-01-1068

[28] F. Piscaglia, "Ongoing and future perspective developments in OpenFOAM," Keynote invited talk at the 4th Annual ESI-OpenCFD OpenFOAM User Conference 2016, Cologne (Germany).

[29] The OpenFOAM Foundation. Free open-source CFD. http://www.openfoam.org.

[30] O. Ubbink, "Numerical prediction of two fluid systems with sharp interfaces," Ph.D. dissertation, Imperial College, University of London, 1997.

[31] H. Weller, "Controlling the computational modes of the arbitrarily structured C grid," Monthly Weather Review, vol. 140, pp. 3220-3234, 2012. http://journals.ametsoc.org/doi/pdf/10.1175/MWR-D-11-00221.1

[32] G. Stiesch, Modeling Engine Spray and Combustion Processes, ser. Heat and Mass Transfer. Springer Berlin Heidelberg, 2003. https://books.google.co.uk/books?id=4A35ABlw1wgC 